\title{
PAPR REDUCTION OF OFDM SIGNAL BY USING COMBINED HADAMARD AND MODIFIED MEU-LAW COMPANDING TECHNIQUES
}

\author{
Mohamed M. El-Nabawy ${ }^{1}$, Mohamed A. Aboul-Dahab ${ }^{2}$ and Khairy El-Barbary ${ }^{3}$ \\ ${ }^{1}$ Electronic and Communication Dept., Modern Academy for Eng. \& Tech in Maadi \\ (M.A.M), Cairo, Egypt \\ ${ }^{2}$ Electronic and Communication Dept., Arab Academy for Science and Technology and \\ Maritime Transport (AAST), Cairo, Egypt \\ ${ }^{3}$ Electronic and Communication Dept., Canal University, Cairo, Egypt
}

\begin{abstract}
Orthogonal frequency division multiplexing (OFDM) is a technique which gives high quality of service $(Q O S)$ to the users by mitigating the fading signals as well as high data rates in multimedia services. However, the peak-to-average power ratio (PAPR) is a technical challenge that reduces the efficiency of $R F$ power amplifiers. In this paper, we propose the combined Hadamard transform and modified meu-law companding transform method in order to lessen the effects of the peak-to-average power ratio of the OFDM signal. Simulation results show that the proposed scheme reduces PAPR compared to other companding techniques as well as the Hadamard transform technique when used on its own.
\end{abstract}

\section{INDEX TERMS}

Orthogonal Frequency Division Multiplexing (OFDM), Cumulative Complementary Distribution Function (CCDF), Peak to Average Power Ratio (PAPR), Bit Error Rate (BER).

\section{INTRODUCTION}

Orthogonal frequency division multiplexing (OFDM) is attractive multicarrier modulation schemes for high bandwidth efficiency and highly strong immunity to multipath fading. Its basic concept is similar to frequency division multiplexing but there is much dissimilarity; under the assumption that the gap between subcarriers is fixed to the inverse of symbol duration, which is divided into $\mathrm{N}$ subcarriers, spectrum overlap but their orthogonal property is remained. Thus high bandwidth efficiency is achieved. By parallel transmitted with $\mathrm{N}$ subcarriers, the symbol duration becomes $\mathrm{N}$ times as long as compared with a single carrier transmission. These allow strong immunity to multipath fading and reduce the complexity of equalizers [1]. Nowadays OFDM have been contained in the IEEE 802.11, IEEE 802.16 wireless broadband access systems, and in digital audio/video broadcasting (DAB/DVB) standard in Europe, etc. On the other hand, the main disadvantage of OFDM signals is their large peak-to-average power ratio (PAPR), which leads to poor power efficiency, and subsequent performance degradation, of the signal transmitted through the power amplifier [2]. 
For signals with a high PAPR, the average input power must be reduced so that the peak power of the input signal is maintained at most at the saturation input level of the high power amplifier (HPA). If the input power is not backed off, signal distortion takes place resulting in out-of-band spectral regrowth [3]. The amount of back-off must be at least equal to the PAPR (unless some kind of pre-distortion technique is applied). However, HPAs are most efficient when they are driven into saturation. Therefore, input power back-off reduces the efficiency of the HPA and this is detrimental to battery low powered devices. As a consequence, many studies have focused on PAPR reduction leading to the development of many schemes to address it such as coding and tone reservation [4][5][6], pre-distortion schemes, clipping [7][8][9], partial transmit sequence (PTS), selected mapping (SLM), interleaving [10][11][12], nonlinear companding transforms [13][14], Hadamard transforms [15][16] and other techniques etc. Of all those PAPR reduction systems, the easiest system is to use the clipping process. Nevertheless, using clipping processing leads to both in-band and out-of-band distortions and further causes an increase in the BER of the system. Another way of approach is the use of companding techniques which give better performance than clipping techniques because the inverse companding transform (expanding) is applied on the receiver end to reduce the distortion of signals. The use of Hadamard transform may reduce PAPR of OFDM signal while the error possibility of system does not increase [17].

In this paper, an efficient technique for reducing PAPR is introduced. It is based upon joint modified $\mu$-Law companding and Hadamard transform method. This paper is organized as follows: The PAPR problem in OFDM systems is presented in section 2 . The modified $\mu$-law companding transform and Hadamard transform are introduced in sections 3 and 4. In section 5, a description of a PAPR reduction scheme that combines the modified $\mu$-law companding transform and Hadamard transform is described. Simulation results are reported in section 6 and section 7 presents the conclusion.

\section{SYSTEM MODEL}

In this section, the basics of OFDM transmitters and the PAPR definition are reviewed. Consider an OFDM consisting of $\mathrm{N}$ subcarriers. Let a block of $\mathrm{N}$ symbol $\mathrm{x}=\left\{\mathrm{X}_{\mathrm{k}}, \mathrm{k}=0,1,2, \ldots, \mathrm{N}-1\right\}$ is formed with each symbol modulating one of a set of subcarriers $\left\{\mathrm{f}_{\mathrm{k}}, \mathrm{k}=0,1,2, \ldots, \mathrm{N}-1\right\}$. the $\mathrm{N}$ subcarriers are chosen to be orthogonal, that is, $f_{k}=k \Delta f$, where $\Delta f=1 /(N T)$ and $T$ is the original symbol period. Therefore, the complex baseband OFDM signal can be written as

$$
x(t)=\frac{1}{\sqrt{N}} \sum_{k=0}^{N-1} X_{k} e^{j 2 \pi f_{k} t}, \quad 0 \leq t \leq N T
$$

In general, the PAPR of an OFDM signal, $x(t)$, is defined as the ratio period between the maximum instantaneous power and its average power during an OFDM symbol

$$
P A P R=\frac{\max _{0 \leq t \leq N T}\left[|x(t)|^{2}\right]}{1 / N T \int_{0}^{N T}|x(t)|^{2} d t}
$$

Reducing the $\max |x(t)|$ is the principle goal of PAPR reduction techniques. In practice, most systems deal with a discrete-time signal, therefore, we have to sample the continuous time signal $\mathrm{x}(\mathrm{t})$. 
To better approximate the PAPR of continuous-time OFDM signals, the OFDM signals samples are obtained by L times oversampling. By sampling $x(t)$ defined in Eq. (1), at frequency $f_{S}=L / T$, where $\mathrm{L}$ is the oversampling factor, the discrete-time OFDM symbol can be written as

$$
x(n)=\frac{1}{\sqrt{N}} \sum_{k=0}^{N-1} X_{k} e^{j \frac{2 \pi}{N L} k n}, 0 \leq n \leq N L-1
$$

Equation (2) can be implemented by using a length (NL) IFFT operation. The new input vector X is extended from original $\mathrm{X}$ by using the so-called zero-padding scheme, i.e. by inserting $\square \mathrm{L}$ $-1 \square \mathrm{N}$ zeros in the middle of $\mathrm{X}$. The PAPR computed form the L-times oversampled time domain OFDM signal samples can be defined as

$$
\text { PAPR }=10 \log \frac{\max n \leq N L 1\left[|x(n)|^{2}\right]}{E\left[|x(n)|^{2}\right]}
$$

However, the PAPR does not increase significantly after $\mathrm{L}=4$. So as to be away from aliasing the out-of-band distortion into the data bearing tones and in so as to accurately have the description of the PAPR an oversampling factor $\mathrm{L} \geq 4$ is necessarily required.

\section{MODIFIED $\mu$-LAW COMPANDING TRANSFORM}

In this section, we simply review the companding techniques [7][8][15][18] of OFDM signal PAPR reduction. This transform reduces the PAPR of OFDM signals by amplifying the small signals and shortening the big signals. In companding, the OFDM signal is compressed at the end of the transmitter after the IFFT process and expanded at the receiver end prior to the FFT process. In this companding method, the compressor characteristic is piecewise, and it consists of a linear segment for low level inputs and a logarithmic segment for high level inputs. The A-law compressor characteristics for different values of $A$ are shown in Figure 1. Corresponding to $A=1$, we notice that the characteristic is linear (no compression) which corresponds to a uniform quantization. A-law has mid riser at the origin. Hence it contains non-zero value. The practically used value of "A" is 87.6. The A-law companding is used for PCM telephone systems [19].

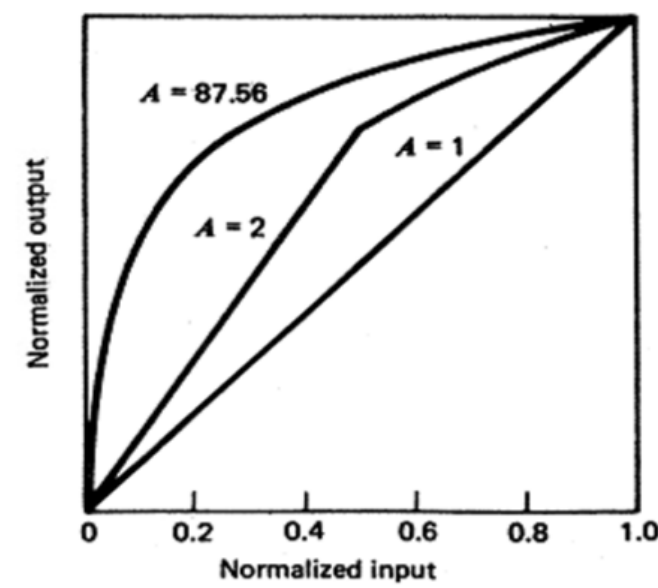

Figure 1 :Characteristics of A-law Compressor

The linear segment of the characteristic is for low level inputs while the logarithmic segment is concerned with high level inputs. It is mathematically expressed as, 


$$
y(x)- \begin{cases}y_{\max } \frac{A \frac{|x|}{x_{\max }}}{(1+A)} \operatorname{sgn}(x) ; \quad 0<\frac{|x|}{x_{\max }} \leq \frac{1}{A} \\ y_{\max } \frac{\left[1+A \frac{|x|}{x_{\max }}\right]}{(1+A)} \operatorname{sgn}(x) ; \frac{1}{A}<\frac{|x|}{x_{\max }} \leq 1\end{cases}
$$

Where $\mathrm{x}=$ input signal, $\mathrm{y}=$ output $\operatorname{signal}, \operatorname{Sgn}(\mathrm{x})=$ sign of the input $(+$ or -$),|\mathrm{x}|=$ absolute value (magnitude of $\mathrm{x}$ ).

In the $\mu$-law companding, the compressor characteristic is piecewise, and is made up of a linear segment for low level inputs and a logarithmic segment for high level inputs. The $\mu$-law compressor characteristics for different values of $\mu$ are shown in figure 2 . The higher the value of " $\mu$ ", the greater the compression. Corresponding to $\mu=0$, we observe that the characteristic is linear (no compression) which corresponds to a uniform quantization. $\mu$-law has mid tread at the origin. Hence it contains zero value. The practically used value of " $\mu$ " is 255 . The $\mu$-law companding is used for speech \& music signals [19].

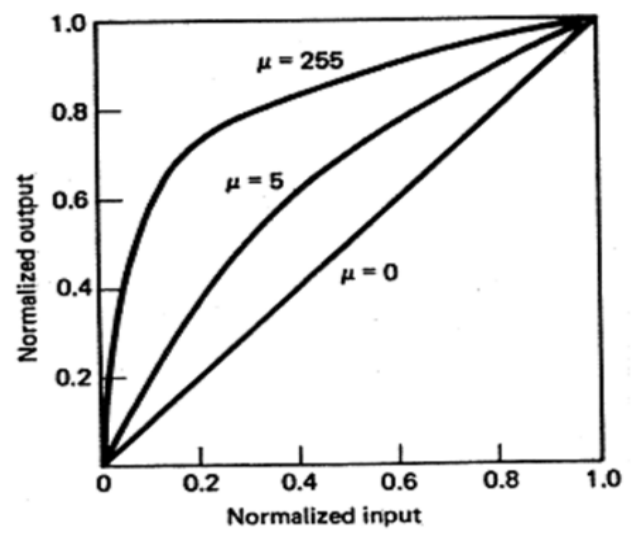

Figure 2 :Characteristics of $\mu$-law Compressor

It is mathematically expressed as,

$$
\frac{C|X|}{x_{\max }}=\frac{\ln \left[1+\mu \frac{|x|}{x_{\max }}\right]}{\ln [1+11]} ; \quad 0<\frac{|x|}{x_{\max }} \leq 1
$$

Where $\mathrm{x}=$ amplitude of the input signal at a particular instant of time, $x_{\max }=$ maximum uncompressed analog input amplitude, $\mathrm{C}|\mathrm{x}|=$ compressed output amplitude, and $|\mathrm{x}|$ absolute value (magnitude of $\mathrm{x}$ ). Note that $\mu$ is the parameter used to define the amount of compression or compression factor.

The first difference between A and $\mu$-law is the dynamic range of the output. $\mu$-law has a larger dynamic range than A-law. Dynamic range is fundamentally the ratio between the quietest and loudest sound that can be represented in the signal. The downside of having a higher dynamic range is greater distortion of small signals; in other words, for soft sound input A-law sounds better than $\mu$-law. The Compression is performed according to the well-known modified $\mu$-Law viz. After companding, the signal now become 
International Journal of Computer Networks \& Communications (IJCNC) Vol.6, No.5, September 2014

$$
\begin{aligned}
& T(n)=C\{x(n)\}=\left(P R X_{\text {peak }}\right) \frac{\left.\log \left(1+\mu_{\text {PRX }}\right) \frac{|x|}{\log (1+\mu)}\right)}{\text { And }} \operatorname{PR}=\frac{V}{x_{\text {peak }}} \\
& \text { Ann }(x)
\end{aligned}
$$

Where PR is peak ratio, $\mathrm{V}$ is the peak amplitude of the input and output signals specified for the $\mu$-Law compander, $x_{\text {peak }}$ is the peak amplitude of the actual signal to be companded $x$ is the instantaneous amplitude of the input signal. The companding transform has to satisfy the following two conditions in particular:

$$
\begin{aligned}
& \text { (1) } \mathrm{E}\left(|T(n)|^{2}\right) \approx \mathrm{E}\left(|x(n)|^{2}\right) \\
& \text { (2) }|T(n)| \geq|x(n)| \text { when }|x(n) \leq V| ; \\
& |T(n)| \geq|x(n)| \text { when }|x(n) \leq V| .
\end{aligned}
$$

Decompression is simply the inverse of equation (5), the expanding equation is

$$
y(n)=C^{-1}\{r(n)\}=\left(\frac{P R X_{\text {Peak }}}{\mu}\right)\left(10^{\frac{|x|(1+\mu)}{P R X_{\text {peak }}}-1}\right) \operatorname{sgn}(x)
$$

\section{HADAMARD TRANSFORM}

Park et.al, have suggestions of in [16] a system for PAPR reduction in OFDM transmission using Hadamard transform. The Hadamard transform scheme may reduce the occurrence of the high peaks compared to the original OFDM system. The idea of using the Hadamard transform is to reduce the autocorrelation of the input sequence to reduce the PAPR problem without needing the transmission of any side information to the receiver. In this section, we shorty review Hadamard transform. We assume $\mathrm{H}$ is the Hadamard transform matrix of $\mathrm{N}$ orders, and that the Hadamard matrix is a standard orthogonal matrix. Every element of the Hadamard matrix is either 1 or -1 . The Hadamard matrix of 2 orders is stated by

$$
H_{2}-\frac{1}{\sqrt{2}}\left[\begin{array}{cc}
1 & 1 \\
1 & -1
\end{array}\right]
$$

Hadamard matrix of $2 \mathrm{~N}$ order may be constructed by

$$
H_{2 N}=\frac{1}{\sqrt{2 N}}\left[\begin{array}{cc}
H_{N} & H_{N} \\
H_{N} & -H_{N}
\end{array}\right]
$$

Where $-H_{N}$ is the complementary of $H_{N}$. Hadamard matrix satisfy the relation

$$
H_{2 N} H_{2 N}^{T}=H_{2 N}^{T} H_{2 N}=I_{2 N}
$$

Where $H_{2 N}^{T}$ is the transport matrix, $I_{2 N}$ is the unity matrix of $2 \mathrm{~N}$ order. Note that the Hadamard can be implemented by using a butterfly structure as in FFT. This means that applying Hadamard transform does not require the extensive increase of system complexity. 
After the sequence $x=\left[x_{1} x_{2} \ldots \ldots \ldots x_{N}\right]^{T}$ is transformed by a Hadamard matrix of $\mathrm{N}$ order, the new sequence is

$$
Y=H X
$$

\section{PROPOSED SCHEME}

The proposed system block diagram is shown in Figure (3). The modification in this system from the classical OFDM system is the combination of the companding transform and the Hadamard transform. These two modifications are added to the OFDM system as shown in Figure (3).

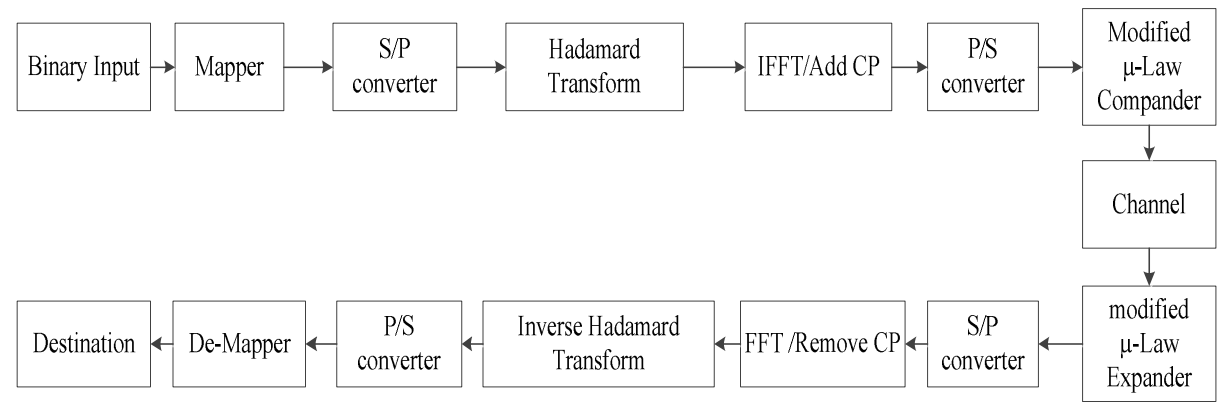

Figure 3: Block diagram of OFDM system with proposed PAPR reduction technique

The signal output of the Hadamard transform unit is given by (14). After the IFFT module the signal will be y=IFFT(Y), where $y=\left[\begin{array}{llll}y(1) & y(2) \ldots \ldots . . . y(N)\end{array}\right]^{T}$. The signal will pass through the P/S converter after which it is applied to the modified $\mu$-law compander. The output of the compander is given by $T(n)=C\{y(n)\}$. The compander signal passes through the AWGN channel. At the receiver, the received signal is transform by modified $\mu$-law expander. The output of expander is given by $\hat{y}(n)=C^{-1}\{r(n)\}$. The expander signal will pass through the $\mathrm{S} / \mathrm{P}$ converter after which it is applied to FFT module. The output signal of FFT module is given by $\hat{Y}=F F T(\hat{y})$. The signal output from the inverse Hadamard transform is given by $\hat{X}=H^{T} \hat{Y}$ then the signal $\hat{X}$ is de-mapped to bit stream.

\section{SIMULATION RESULTS}

In this section, the simulation results of the proposed scheme are presented. These results are the complementary CDF (CCDF) performance and the bit error rate (BER) achieved. The CCDF performance of the proposed scheme is evaluated by two steps of simulations. Simulations are done on the Matlab package. Simulations are made using of QPSK as a modulation scheme with 64 subcarriers and an additive white Gaussian noise (AWGN) channel.

The cumulative distribution function (CDF), a widely used parameter, is employed to measure how efficient PAPR techniques are. The CDF of the amplitude of a signal sample is given by

$$
F(z)=1-\exp (z)
$$

However, the complementary CDF (CCDF) is used instead of the CDF, which helps us to measure the probability that the PAPR of a certain data block exceeds the given threshold. The 
International Journal of Computer Networks \& Communications (IJCNC) Vol.6, No.5, September 2014

CCDF of the PAPR of the data block is desired in our case to compare outputs of various reduction techniques. It is given by

$$
P(P A P R>z)=1-P(P A P R>z)=1-(1-\exp (-z))^{N}
$$

In the first simulation, the value of $\mathrm{PR}=1$ while the companding factor $\mu$ has four different values. First, when $\mu=1$ the performance of the proposed scheme provides better performance than that of the simple companding, Hadamard or classical OFDM. at $\mathrm{CCDF}=10^{-4}$, Figure (4-a) illustrates this achievement where the PAPR of the proposed scheme is almost $2.8 \mathrm{~dB}$ smaller than that of classical OFDM scheme. The Hadamard matrix transform will reduce 1.2 dB PAPR compared to that of classical OFDM signal.

Second when $\mu=2$ the PAPR is reduced by $3.4 \mathrm{~dB}$ compared to classical OFDM as shown in figure (4-b).Third when $\mu=5$ Fourth when $\mu=10$ this gets a reduction value of $5.8 \mathrm{~dB}$ as shown in Figure (4-c) and Figure (4-d) respectively.

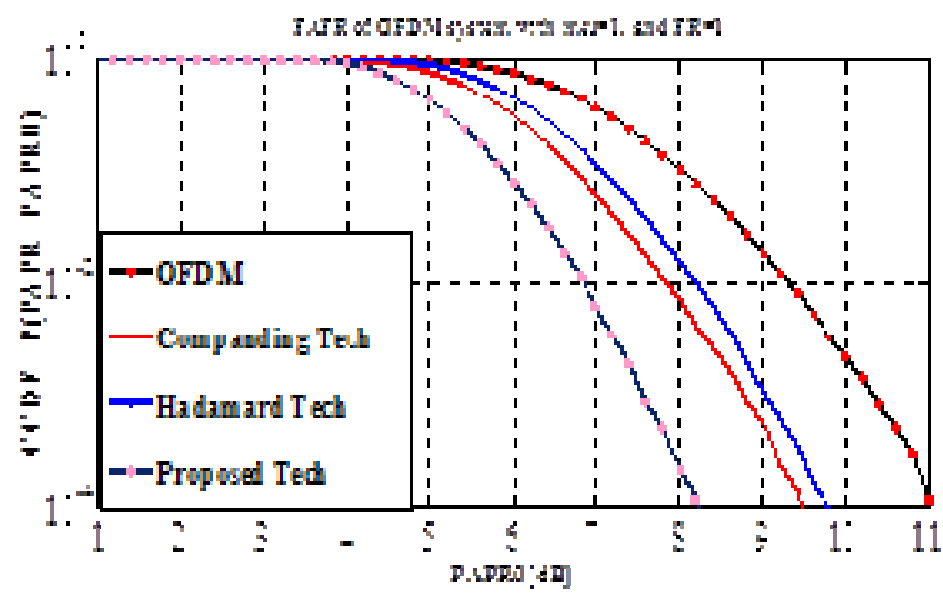

(a)

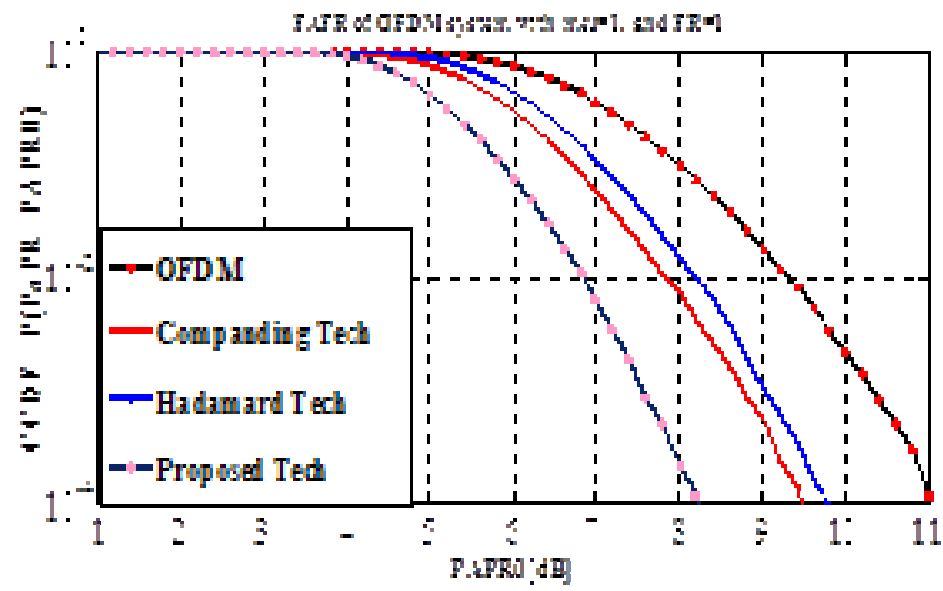

(b) 
International Journal of Computer Networks \& Communications (IJCNC) Vol.6, No.5, September 2014

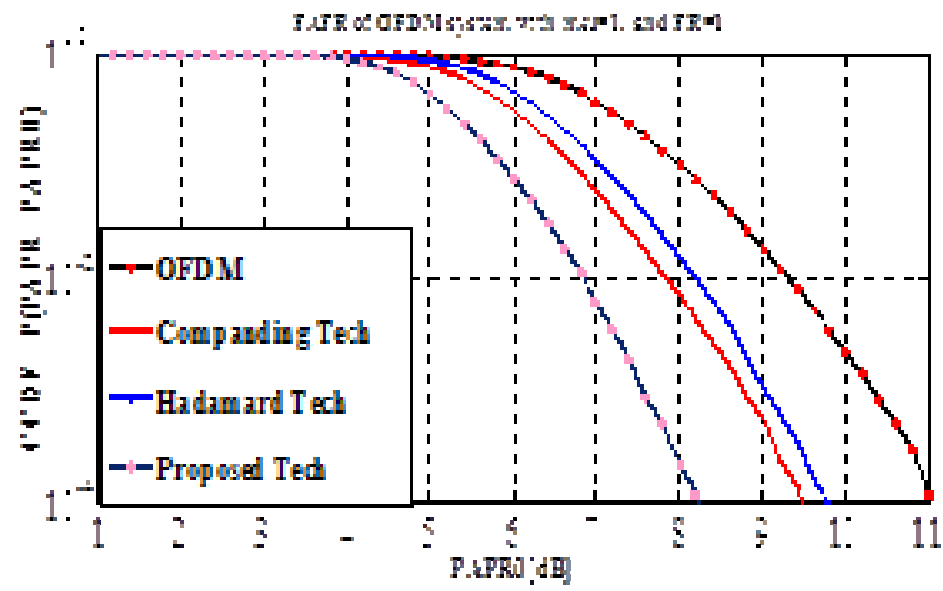

(c)

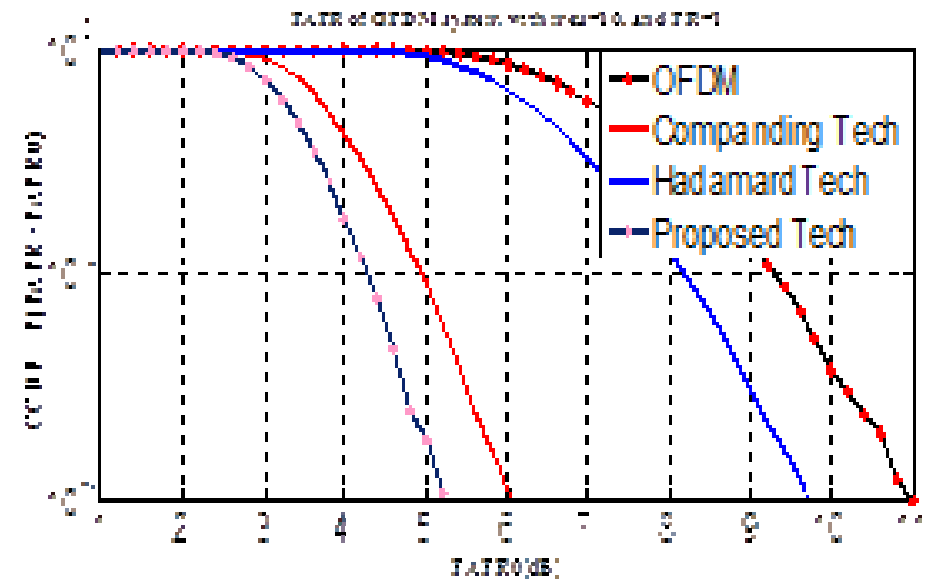

(d)

Figure 4:Comparison of PAPR for OFDM, Hadamard transform techniques, companding techniques, and proposed scheme at $P R=1$ and different value $\mu$ (a) $\mu=1$, (b) $\mu=2$, (c) $\mu=5$, (d) $\mu=10$.

In the second simulation the value of peak ratio $P R=2$ with the same four values of used in the first simulation., Figure 3 show that the proposed scheme is lower than classical OFDM system and Hadamard and modified $\mu$-law companding techniques . Although the reduction of this simulation is low but it is not as low as the first simulation. This is obvious in Figure (5-a) the PAPR of proposed scheme is almost $2.2 \mathrm{~dB}$ smaller than that of classical OFDM signal. This value of reduction is less than the previous by $0.6 \mathrm{~dB}$. 
International Journal of Computer Networks \& Communications (IJCNC) Vol.6, No.5, September 2014

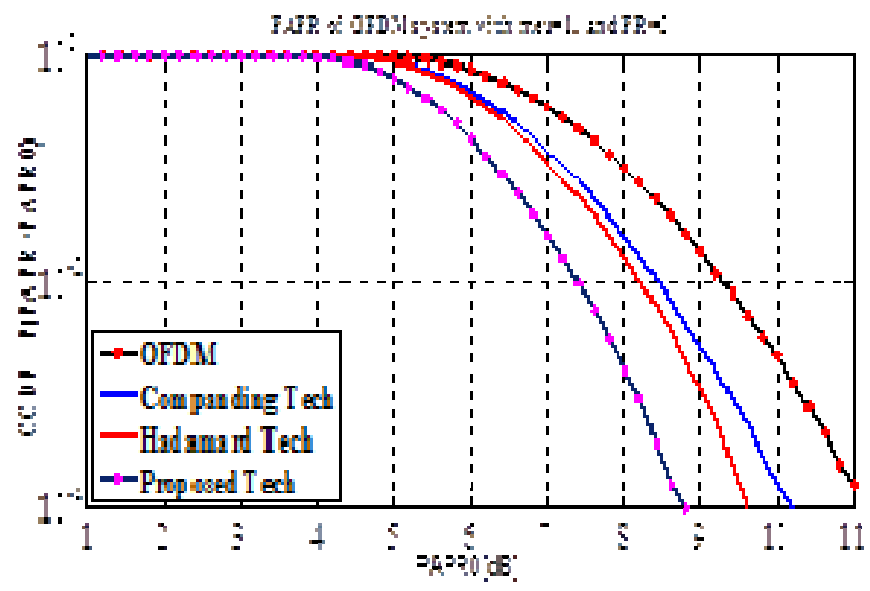

(a)

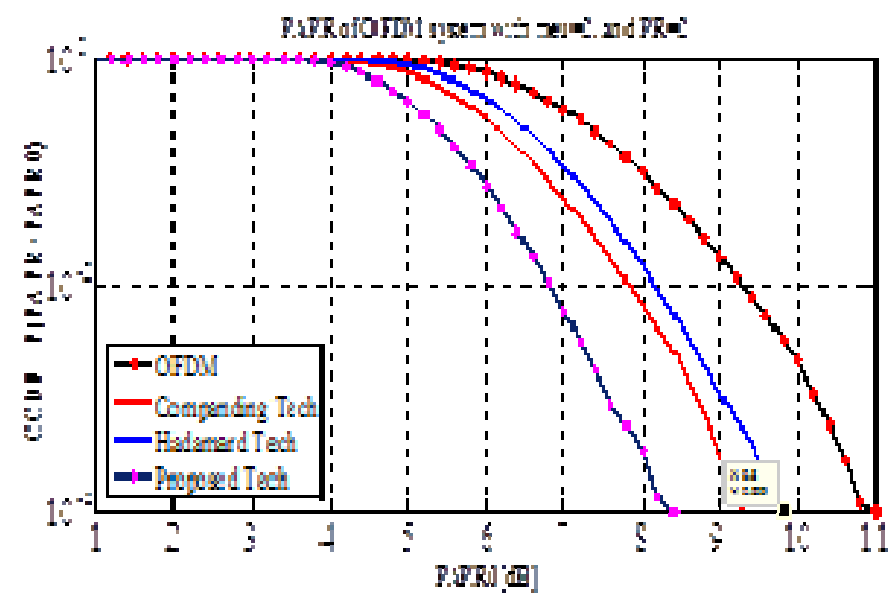

(b)

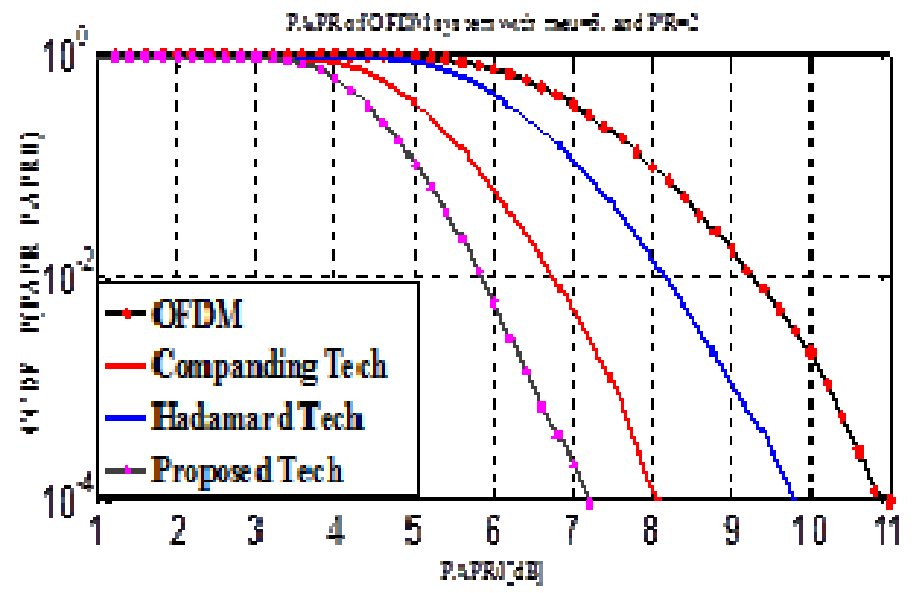

(c) 


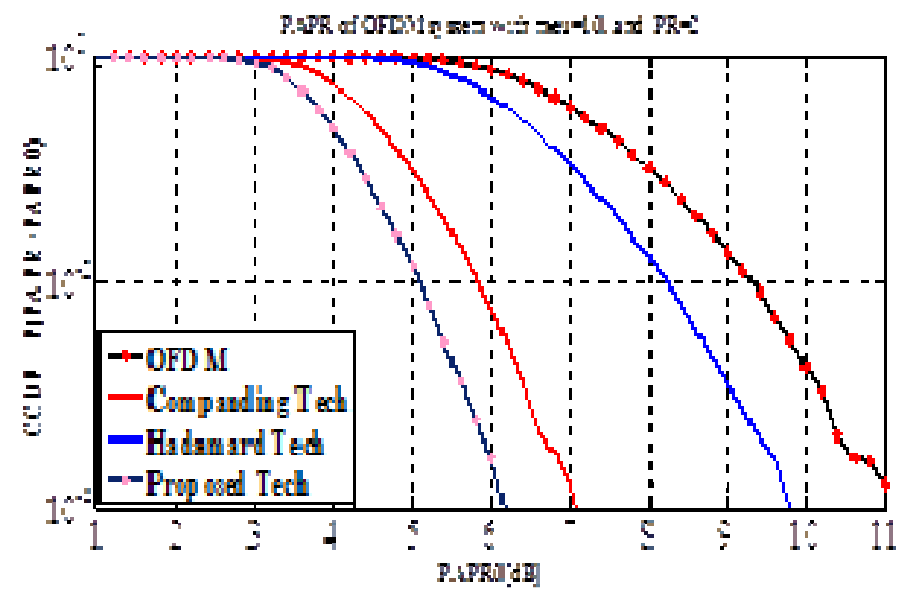

(d)

Figure 5:Comparisons of PAPR for OFDM, Hadamard transform techniques, companding techniques, and proposed scheme at $P R=2$ and different value $\mu$ (a) $\mu=1$, (b) $\mu=2$, (c) $\mu=5$, (d) $\mu=10$.

Table I summarize the values of PAPR for the classical OFDM system, Hadamard transform, companding technique and the proposed scheme.

As an example for $\mathrm{PR}=1$ and $\mu=5$, the PAPR of the proposed scheme, companding technique, Hadamard transform, and OFDM system is equal 6.1, 7.1, 9.8, $11 \mathrm{~dB}$ respectively. We can see the amount of reduction of OFDM system, Hadamard transform, and companding technique with respect to proposed scheme is equal $4.9,3.7,1 \mathrm{~dB}$ respectively. From table- 1 we can see that at different values of $\mu$, the PAPR of proposed scheme is less than that of other techniques, But the value of reduction at $P R=1$ is greater than of the value at $P R=2$.

TABLE I. VALUE OF PAPR0 IN dB AT CCDF $=10^{-4}$ FOR DIFFERENT PAPR REDUCTION TECHNIQUES.

\begin{tabular}{|c|c|c|c|c|c|c|}
\hline \multirow{2}{*}{$\begin{array}{c}\text { Tech } \\
\mu\end{array}$} & \multicolumn{2}{|c|}{ Proposed } & \multicolumn{2}{c|}{ Companding } & \multirow{2}{*}{ Hadamard } & OFDM \\
\cline { 2 - 5 } 1 & $\mathrm{PR}=1$ & $\mathrm{PR}=2$ & $\mathrm{PR}=1$ & $\mathrm{PR}=2$ & & 11 \\
\hline 2 & 7.2 & 8.8 & 9.5 & 9.6 & 9.8 & 11 \\
\hline 5 & 6.1 & 7.2 & 7.1 & 8.1 & 9.8 & 11 \\
\hline 10 & 5.2 & 6.2 & 6 & 7.1 & 9.8 & 11 \\
\hline
\end{tabular}

Figure (6) presents the BER performance over AWGN channel for OFDM system, Hadamard transform, companding technique, and proposed scheme. The companding and proposed scheme uses two different values of PR. In Figure (6-a) and Figure (6-b) we can see that the performance of proposed system did not degrade compared to other systems. While in Figure (6-c) and Figure (6-d) we can see at the large value of companding factor $\mu$, the performance of BER is degraded. But at the large value of PR this degraded can be minimized. 
International Journal of Computer Networks \& Communications (IJCNC) Vol.6, No.5, September 2014

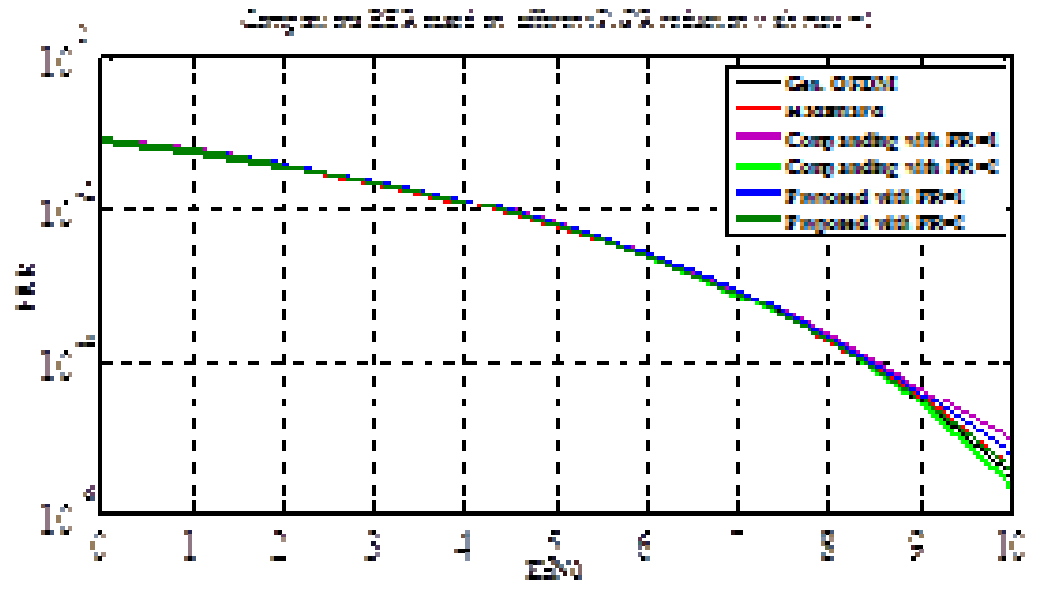

(a)

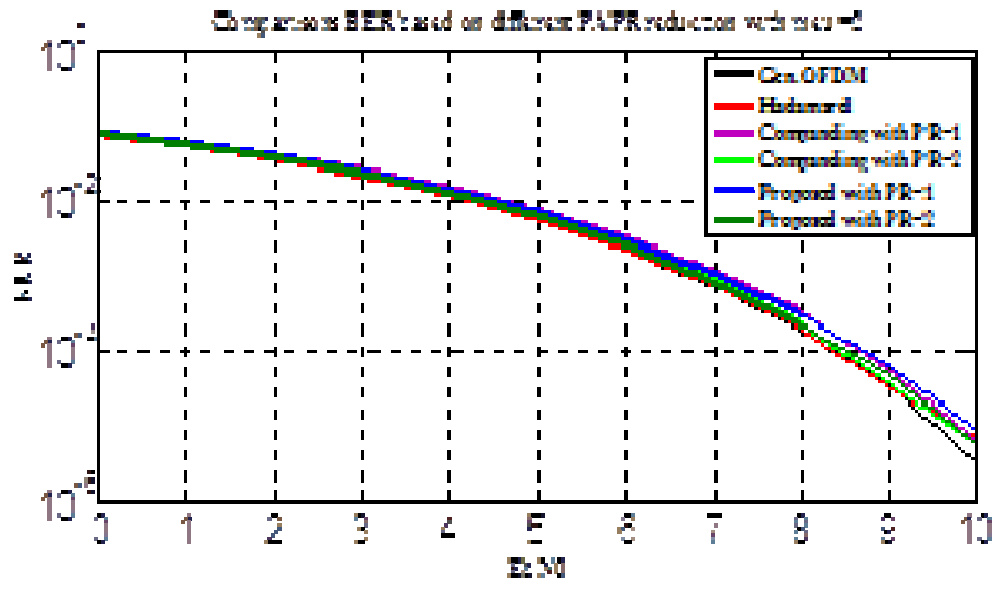

(b)

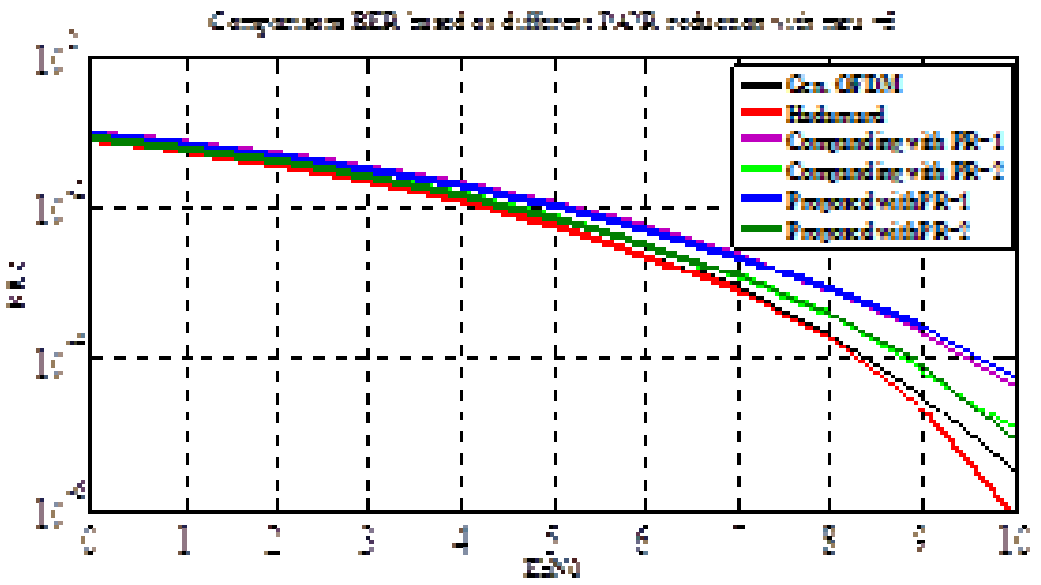

(c) 
International Journal of Computer Networks \& Communications (IJCNC) Vol.6, No.5, September 2014

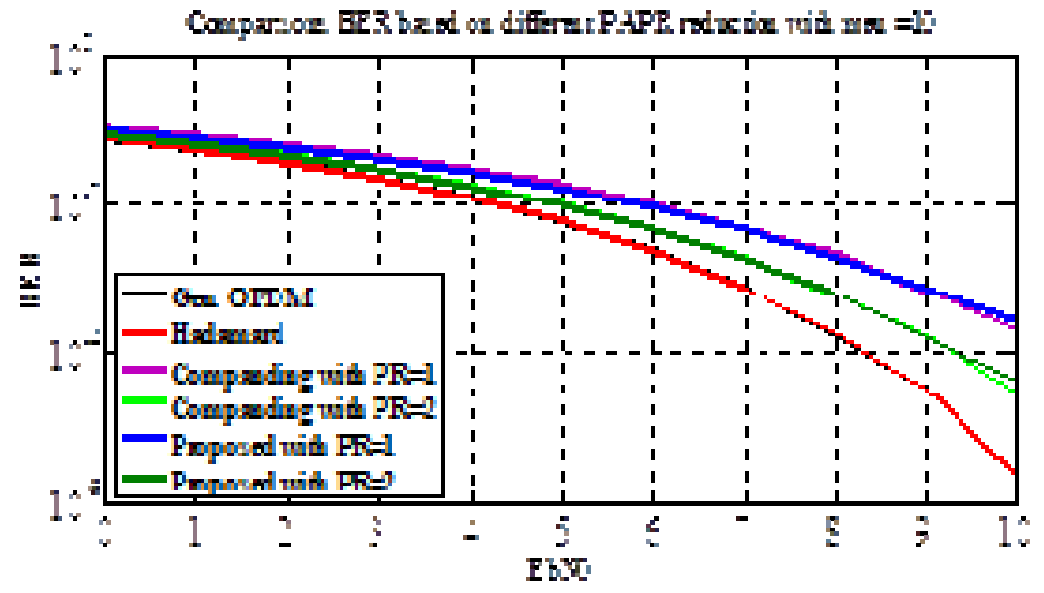

(d)

Comparisons of BER for OFDM, Hadamard transform techniques, companding techniques, and proposed scheme at $P R=1$ and 2 and different value $\mu$ (a) $\mu=1$, (b) $\mu=2$, (c) $\mu=5$, (d) $\mu=10$.

Table II summarize the value of $E_{b} / N_{0}$ in $\mathrm{dB}$ for different value of $\mu$ and PR for different techniques of PAPR reduction at the BER $=10^{-4}$. The table shows the BER performance at low values of $\mu$ did not degraded that mean higher value of PR will not increase the performance of the BER. But at the large value of $\mu$ you must use large value of PR to upgrade value of BER. As example at $\mu=2$ the value of $E_{b} / N_{0}$ approximately don't changed for different techniques and PR. But at $\mu=10$ the value of $E_{b} / N_{0}$ for OFDM system, Hadamard is equal. But for proposed system the value of $E_{b} / N_{0}$ is changed by $2.1 \mathrm{~dB}$ at $\mathrm{PR}=1$, this value is large, we can upgrade this by increase value of PR. At $\mathrm{PR}=2$ the changed of $E_{b} / N_{0}$ w.r.t OFDM system at $\mathrm{BER}=10^{-4}$ equal $1 \mathrm{~dB}$. By comparing change in PR modify in degraded of BER.

TABLE II. THE VALUE OF $E_{b} / N_{0}$ IN dB FOR DIFFERENT VALUE OF PR \& DIFFERENT TECHNIQUES OF PAPR REDUCTION AT BER $=10^{-4}$

\begin{tabular}{|c|c|c|c|c|c|c|}
\hline \multirow{2}{*}{\begin{tabular}{c}
\multirow{2}{*}{$\mu$} \\
Tech
\end{tabular}} & \multicolumn{2}{|c|}{ Proposed } & \multicolumn{2}{c|}{ Companding } & \multirow{2}{*}{ Hadamard } & \multirow{2}{*}{ OFDM } \\
\cline { 2 - 5 } & $\mathrm{PR}=1$ & $\mathrm{PR}=2$ & $\mathrm{PR}=1$ & $\mathrm{PR}=2$ & & \\
\hline 1 & 8.4 & 8.4 & 8.4 & 8.4 & 8.4 & 8.4 \\
\hline 2 & 8.6 & 8.4 & 8.6 & 9.3 & 8.4 & 8.4 \\
\hline 5 & 9.5 & 8.8 & 9.5 & 8.8 & 8.4 & 8.4 \\
\hline 10 & 10.5 & 9.4 & 10.5 & 9.4 & 8.4 & 8.4 \\
\hline
\end{tabular}

\section{CONCLUSIONS}

In this paper, a PAPR reduction scheme which is basically based on combination of Hadamard transform and modified $\mu$-law companding techniques is proposed. This modified $\mu$-law profile is based upon the peak ratio (PR). The PAPR reduction performance and BER performance are evaluated through computer simulation. Two different simulations have been carried out for the proposed scheme. The first simulation is made for the case $\mathrm{PR}=1$ and $\mu$ have four different values 
which are the second simulation uses the same values of $\mu$ but $P R=2$. Simulation results show that the PAPR reduction performance is improved compared to only using companding transforms. On the other hand, the BER of the system using the proposed PAPR reduction scheme does not degrade at small values of the companding factor, $\mu$. At large values of $\mu$, however, the BER performance degrades. This issue can be solved by increasing the value of the peak ratio, PR. As the PR increases, the PAPR reduction decreases; regardless, the proposed scheme still remains better than the companding techniques, Hadamard transform, and OFDM system.

\section{REFERENCES}

[1] A. R. Bahai and B. R. Saltzberg, "Multi-Carrier Digital Communications: Theory and Applications of OFDM,” Kluwer Academic/Plenum Publishes, New York, NY, USA, 1999.

[2] H. Rohling, T. May, K. Bruninghaus, and R. Grunheid, "Broadband OFDM radio transmission for multimedia applications,"Proc. IEEE, vol.87,pp.1778-1789,Oct.1999.

[3] Marilynn P. Wylie-Green, etal. ," Introduction to CPM-SC-FDMA: A Novel Multiple-Access PowerEfficient Transmission Scheme”, IEEE TRANSACTIONS ON COMMUNICATIONS, VOL. 59, NO. 7, JULY 2011.

[4] J. A. Davis and J. Jedwab, "Peak-to-mean power control in OFDM Golay complementary sequences," IEEE Trans. Inf. Theory, vol. 45,pp. 2397-2417, Nov. 1999.

[5] B. S. Krongold and D. L. Jones, "An active-set approach for OFDM PAR reduction via tone reservations," IEEE Trans. Signal Process., vol. 52,pp. 495-509, Feb. 2004.

[6] A. D. S. Jayalath and C. R. N. Athaudage, "On the PAR reduction of OFDM signals using multiple signal representation," IEEE Commun.Lett., vol. 8, pp. 425-427, July 2004

[7] V. Cuteanu, A. Isar, "PAPR reduction of OFDM signals using hybrid clipping-companding scheme with sigmoid functions", International Conference on Applied Electronics, pp. 75-78, September 2011.

[8] A. N. D' Andrea, V. Littici, and R. Reggiannini, "Nonlinear predistortion of OFDM signals over frequency-selective fading channels," IEEETrans. Commun., vol. 49, pp. 837-43, May 2001.

[9] J. Armstrong, "Peak-to-average power reduction for OFDM by repeated clipping and frequency domain filtering," IEE Electron. Lett., vol. 38,p. 246-247, Feb. 2002.

[10] L. Wang and J. Liu, "PAPR Reduction of OFDM Signals by PTS With Grouping and Recursive Phase Weighting Methods", IEEE Transactions on Broadcasting, vol. 57, no.2, pp. 299-306, June 2011.

[11] T. Jiang and Y. Imai, "An overview: peak-to-average power ratio reduction techniques for OFDM signals,” IEEE Trans. On Wireless Communications, June 2008, pp. 56-57

[12] S.H. Han, J. H. Lee, "An overview of peak-to-average power ratio reduction techniques for Multicarrier transmission," IEEE Transaction on Wireless Communication, April 2005, pp. 56-65.

[13] X. Huang, etal. "Companding Transform for Reduction in Peak-to-Average Power of OFDM signals", IEEE transactions on wireless communications, VOL.03, NO.06, Nov,2004,pp. 2030-2039.

[14] X. B. Wang, "Reduction of peak-to-average power ratio of OFDM system using A companding technique," IEEE Transaction on Broadcasting, Vol.45, No.3, Sep 1999, pp.303-307.

[15] Z. Wang, S. Zhang, "PAPR reduction of OFDM signal by using Hadamard transform in companding techniques," IEEE International Conference Communications, pp.320-323, Nov 2010.

[16] M. Park, etal., "PAPR reduction in OFDM transmission using Hadamard transform", IEEE International Conference o Communications, Vol.1, Jun 2000, pp.430-433.

[17] X. Zhu, etal. ,"Reducing the peak-to-average power ratio using unitary matrix transformation. IET Communications, 2009,3(2),pp.161-171.

[18] A.Vallavaraj, etal. "An evaluation of modified m-Law companding to reduce the PAPR of OFDM systems”, Int. J.Electron.Commun.(AEU“) 64 (2010) 844-857.

[19] Vishwajit N. Sonawane, etal. ," Comparative Analysis between A-law \& $\mu$-law Companding Technique for PAPR Reduction in OFDM", International Journal of Advanced Research in Computer and Communication Engineering Vol. 2, Issue 5, May 2013. 


\section{Authors}

Mohamed M. El-Nabawy received the B.S degree in Electronics Engineering and communications Technology (with average grade excellent with honor degree) from Modern Academy for Eng. \& Tech. (M.A.M), Cairo, Egypt, in 2007. Currently pursuing the Master degree \& working as Teaching Assistant in Electronics and communications engineering in M.A.M., His Research area includes Wireless Communication, Mobile Communication, Information \& Coding Theory, etc.

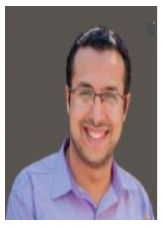

Mohamed A. Aboul-Dahab received the B.S.degrees in communication engineering from Faculty of Engineering, Cairo Univ., in 1973. He received the M.Sc. and Ph.D. degrees in Communications Engineering from Alexandria Univ., Egypt in 1980 and 1986 respectively. $\mathrm{He}$ is a professor in the Electronics and Communications Engineering department since 1999. He has been the Head of the Electronics and Computer Engineering Department, College of Engineering and Technology (Alexandria Campus), Arab Academy for Science and Technology and Maritime Transport, AASTMT (1995-2002), and the Dean of College

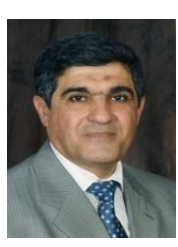
of Engineering and Technology (Cairo Campus), AASTMT (2002-2008) .He has 37 years of teaching experience for the undergraduate students and postgraduate. He has supervised many undergraduate projects and many master and Ph.D. theses in AASTMT and other universities. His research activities are in the field of antennas, digital communication and satellite communication.

K.A. ElBarbary received the B. S. degree (with Average Grade Very Good) in Electric Engineering from Military Technical College, Egypt, the M.S. degree in Electric Engineering from Military Technical College, Egypt, the Ph.D. degree in Communications from George Washington University, Washington DC, USA, in 1981, 1986 and 1993, respectively. He was faculty member at Military Technical College, Egypt in the Electric Engineering Department (Chair of communications and Electronic warfare) (1994-2010). there he became a professor of Electric Engineering(2009), Head of Electronic Warfare Engineering Department (2000), and Head of Electrical Engineering Department (2009). He has been a professor of signal and systems and Communications at Suez Canal University, Egypt in Electric Engineering Department. Currently he is the Head of Electric Engineering Department, Suez Canal University, Egypt. His research interests include Statistical signal and array processing and related applications in communications, radar, Electronic Intelligence, Multi user Detectors and wide band communication systems. 